\title{
Catalysis on Pristine 2D Materials via Dispersion and
}

\section{Electrostatic Interactions}

\author{
Amir Karton \\ School of Molecular Sciences, The University of Western Australia, Perth, WA 6009, Australia
}

\begin{abstract}
:
Shape complementarity between catalyst and transition state structure is one of the cornerstones of chemical catalysis. Likewise, noncovalent interactions play a major role in catalysis. It has been predicted computationally, and recently confirmed experimentally [Kroeger, A. A.; Hooper, J. F.; Karton, A. ChemPhysChem, 2020, in press, DOI: $10.1002 / \mathrm{cphc} .202000426]$, that pristine graphene can efficiently catalyze chemical processes via $\Pi$ interactions and shape complementarity. Here we show that other two-dimensional materials with different electronic structures and chemical compositions (h-BN and graphane) can also catalyze chemical processes that proceed via planar transition state structures. These include the bowl-to-bowl inversions in corannulene and sumanene and the rotation about the $\mathrm{C}-\mathrm{C}$ bond in substituted biphenyls. This catalytic activity is achieved through shape complementarity between planar nanomaterial and planar transition state structure, enabling disproportionate stabilization of the transition state structures over the non-planar reactants and products. A DFT-based energy decomposition analysis shows that this catalytic activity is mainly driven by dispersion and electrostatic forces, which together outweigh the Pauli repulsion term. These findings enrich and expand the concept of catalysis by pristine 2D materials.
\end{abstract}

\section{Introduction}

Two-dimensional (2D) nanomaterials exhibit a range of chemical properties such as large surface areas and ease of functionalization, which make them attractive candidates as heterogeneous catalysts. Indeed, over the past decade, functionalized graphene and its 
derivatives have emerged as versatile heterogeneous and solid-state catalysts in various areas including photo-, electro-, transition-metal, and metal-free catalysis. . $^{1,2,3,4,5,6,7,8,9,10,11}$ In these graphene-based catalysts, however, catalysis does not occur at the surface of pristine graphene but at chemically modified or functionalized sites (e.g., defects, vacancies, edges, heteroatom dopants, adatoms adsorbed on graphene, and functional groups). Thus, in these functionalized nanomaterials the pristine part of graphene does not play a leading role in catalysis, but more a role of a catalyst support. A potential limiting factor in the application of such functionalized graphene-based catalysts is the challenges associated with controlling the uniform distribution of defects, vacancies, dopants, adatoms, and functional groups.

Van der Waals (vdW) interactions play a prominent role in supramolecular chemistry and nanomaterials. ${ }^{12,13,14,15,16}$ Of particular importance to the present work is the ubiquitous role of $\mathrm{vdW}$ interactions in chemical catalysis. ${ }^{16,17,18}$ Likewise, shape complementarity between catalyst and transition state structure (TSS) is one of the cornerstones of chemical catalysis. Pristine graphene has both a 2D planar morphology and interacts with surrounding molecules via vdW interactions. ${ }^{14}$ These two properties make pristine graphene an ideal catalyst for catalyzing chemical processes that proceed via a planar (or nearly planar) transition state structure. Indeed, it has been recently demonstrated, both theoretically and experimentally, ${ }^{19,20,21,22}$ that pristine graphene can efficiently catalyze chemical processes which proceed via a planar (or nearly planar) transition state structure. The first computational investigation used double-hybrid density functional theory (DHDFT) calculations to show that large polycyclic aromatic hydrocarbons $\mathrm{C}_{24} \mathrm{H}_{12}$ and $\mathrm{C}_{54} \mathrm{H}_{18}$ (a.k.a. graphene nanoflakes, GNFs) can efficiently catalyze the bowl-to-bowl inversions in corannulene and sumanene. ${ }^{19}$ Shortly thereafter, Denis used periodic DFT calculations to show that an infinite pristine graphene catalyst reduces the bowl-to-bowl inversion barrier in corannulene and sumanene by over $50 \%$ relative to the uncatalyzed barrier heights. ${ }^{20}$ In this 
context, it should be noted that catalysis of the bowl-to-bowl inversions in corannulene and sumanene via non-bonding intermolecular interactions has been observed experimentally and has attracted considerable attention. ${ }^{23,24}$ Juríček et al. studied the bowl-to-bowl inversion of ethyl-substituted corannulene inside a synthetic cyclophane receptor by variable-temperature, dynamic ${ }^{1} \mathrm{H}$ NMR spectroscopy. ${ }^{23}$ They showed that the cyclophane receptor catalyzes the bowl-to-bowl inversion by destabilizing the reactant and stabilizing the planar transition state structure. It was estimated that the cyclophane catalyst reduces the Gibbs free activation energy for inversion by about $10.5 \mathrm{~kJ} \mathrm{~mol}^{-1}$ relative to the barrier of the uncatalyzed reaction. Shortly thereafter, Jaafar et al. demonstrated via scanning tunneling microscopy that an $\operatorname{Ag}(111)$ surface can assist in the bowl-to-bowl inversion of sumanene adsorbed onto the surface. ${ }^{24}$

In a recent computational investigation, ${ }^{21}$ we used extensive DFT simulations to show that a large GNF (circumcircumcoronene, $\mathrm{C}_{96} \mathrm{H}_{24}$ ) can catalyze the racemization of 1,1'binaphthyl and its synthetically relevant derivative 1,1'-binaphthyl-2,2'-diol (BINOL). This computational prediction has been recently confirmed experimentally through chiral HPLC measurements. ${ }^{22}$ A common motif in the all the above catalytic processes is that noncovalent interactions between the planar catalyst and TSS stabilize the planar TSS to a greater extent than they stabilize the non-planar reactants and products.

In the present work we pose the following question, can other pristine $2 \mathrm{D}$ materials catalyze chemical processes which proceed via a planar TSS? and if so, how do their catalytic efficiencies compare with that of graphene? We focus on 2D materials with significantly different electronic structures and chemical compositions, namely inorganic hexagonal boron-nitride (h-BN) and aliphatic graphane. Like graphene, h-BN has a honeycomb lattice structure; however, whilst graphene involves non-polar homo-nuclear $\mathrm{C}-\mathrm{C}$ bonds, h-BN has highly polar B-N bonds resulting in dramatic differences in their electrical and chemical 
properties. ${ }^{25}$ Graphane, on the other hand, is a fully saturated 2D hydrocarbon containing only $\mathrm{sp}^{3}$ hybridized carbons. In contrast to graphene, graphane has a uniform distribution of vertical $\mathrm{C}-\mathrm{H}$ bonds above and below the molecular plane allowing for $\sigma / \sigma-$ and $\sigma / \pi$ interactions with adsorbed hydrocarbons. Given the significant chemical and structural differences between graphene, h-BN, and graphane it is of interest to see whether h-BN and graphane can catalyze the reactions which graphene has been found to catalyze via $\pi$ interactions. 


\section{Computational Methods}

The geometries and harmonic vibrational frequencies of all structures were obtained from density functional theory (DFT) calculations at the PBE-D3BJ/6-31G(d) level of theory. ${ }^{26,27}$ Empirical D3 dispersion corrections ${ }^{28}$ are included using the Becke-Johnson ${ }^{29}$ damping potential as recommended in ref 30 (denoted by the suffix D3BJ). Zero-point vibrational energies (ZPVEs), enthalpic temperature corrections $\left(H_{298}-H_{0}\right)$, and entropic corrections were obtained from such calculations. The equilibrium structures were verified to have all real harmonic frequencies and the transition state structures to have only one imaginary frequency which corresponds to the expected motion along the reaction coordinate. The connectivities of the transition and equilibrium structures were confirmed via intrinsic reaction coordinate (IRC) calculations. ${ }^{31}$ All geometry optimizations and frequency calculations were carried out using the Gaussian 16 rev. A.03 program suite. ${ }^{32}$

High-level double-hybrid DFT (DHDFT) calculations ${ }^{33}$ were performed using the optimized PBE-D3BJ/6-31G(d) geometries in order to obtain more accurate electronic energies for the equilibrium and transition state structures located along the reaction pathways. We used the recently developed revDSD-PBEP86-D3BJ DHDFT method. ${ }^{34,35}$ This procedure has been found to produce thermochemical and kinetic properties with mean absolute deviations (MADs) below the threshold of 'chemical accuracy' (arbitrarily defined as $\sim 4 \mathrm{~kJ} \mathrm{~mol}^{-1}$ ) from a wide range of accurate experimental and theoretical thermochemical and kinetic determinations. For example, revDSD-PBEP86-D3BJ attains the following MADs for subsets of the GMTKN55 database: ${ }^{36} 2.3$ (thermochemistry), 1.3 (barrier heights), 2.3 (large molecule reactions), 1.9 (conformer energies), 2.4 (intermolecular interactions) kJ mol $^{-1} .{ }^{35}$ The revDSD-PBEP86-D3BJ calculations were carried out in conjunction with the Def2-TZVPP basis set. ${ }^{37}$ We note that due to the large size of the systems considered (e.g., sumanene... graphane system involves 117 carbon and 132 hydrogen atoms) we were unable 
to use a larger basis set in the DHDFT calculations. All the DHDFT calculations were performed using the ORCA 4.2.0 program suite. $^{38}$

To gain qualitative insights into the nature of the noncovalent interactions between the nanomaterial catalyst and substrate, a second-generation energy decomposition analysis $(\mathrm{EDA})^{39,40,41}$ based on absolutely localized molecular orbitals (ALMO) was performed in conjunction with the Def2-SVP basis set. ${ }^{37}$ The EDA calculations were carried out with the $\omega \mathrm{B} 97 \mathrm{M}-\mathrm{V}$ exchange correlation functional. ${ }^{42}$ These calculations were carried out with the QChem 5.2 program suite. ${ }^{43}$

\section{Results and discussion}

High-level quantum chemical calculations (with the revDSD-PBEP86-D3BJ doublehybrid DFT procedure) were performed in order to explore the potential energy surfaces (PESs) for the uncatalyzed and catalyzed bowl-to-bowl inversions in corannulene and sumanene and the rotation about the $\mathrm{C}-\mathrm{C}$ bond in $2,2^{2}$-dimethylbiphenyl. A common feature shared by these reactions is that the substrate has a non-planar $\pi$-system in the reactant and product complexes, whilst it adopts a planar structure in the TSS. As already demonstrated for a graphene catalyst, ${ }^{19,20,21,22} \pi$-interactions between the planar catalyst and planar TSS are stronger than those between the planar catalyst and non-planar reactants and products. Here we will investigate the catalytic properties of other pristine $2 \mathrm{D}$ materials with electronic structures and atomic compositions different from graphene - namely, h-BN and graphane. Following the strategy used in the previous DFT and DHDFT investigations, ${ }^{19,20,21,22}$ the catalysts are modelled using extended fragments of the monolayers, namely $\mathrm{C}_{96} \mathrm{H}_{24}$ for graphene, $\mathrm{B}_{48} \mathrm{~N}_{48} \mathrm{H}_{24}$ for h-BN, and $\mathrm{C}_{96} \mathrm{H}_{120}$ for graphane (Figure 1). 
(a) $\mathrm{C}_{96} \mathrm{H}_{24}$

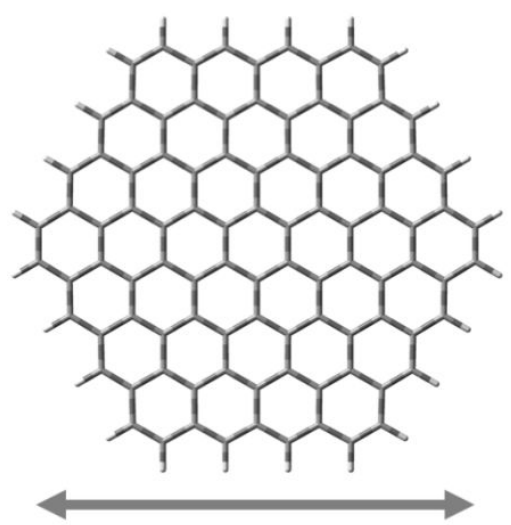

$1.73 \mathrm{~nm}$ (b) $\mathrm{B}_{48} \mathrm{~N}_{48} \mathrm{H}_{24}$

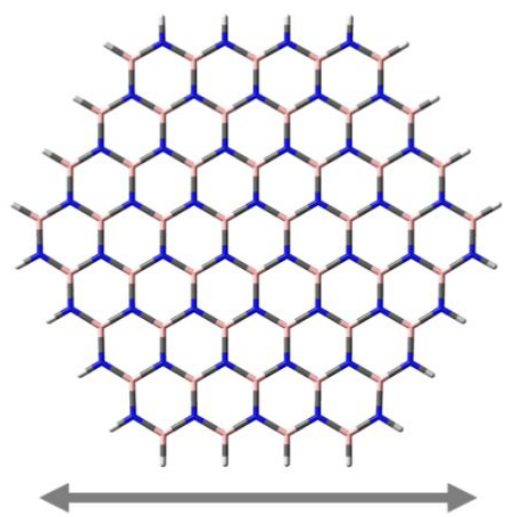

$1.76 \mathrm{~nm}$ (c) $\mathrm{C}_{96} \mathrm{H}_{120}$

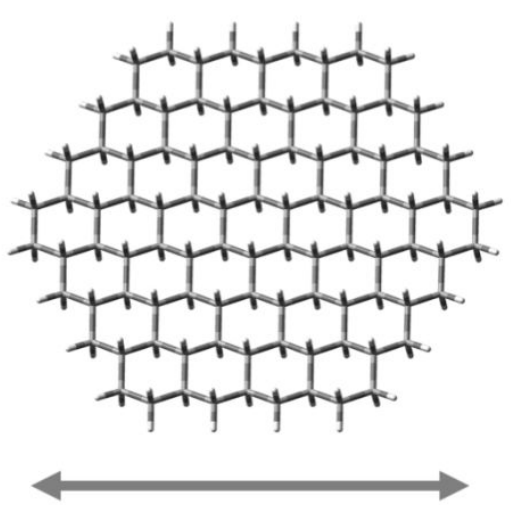

$1.78 \mathrm{~nm}$

Figure 1. Optimized structures of the $2 \mathrm{D}$ catalyst models considered in this work (a) $\mathrm{C}_{96} \mathrm{H}_{24}$ for graphene, (b) $\mathrm{B}_{48} \mathrm{~N}_{48} \mathrm{H}_{24}$ for h-BN, and (c) $\mathrm{C}_{96} \mathrm{H}_{120}$ for graphane. The diameter of the carbon skeleton is given in $\mathrm{nm}$.

\section{Corannulene bowl-to-bowl inversion}

Uncatalyzed reaction. Corannulene is a flexible bowl-shaped polycyclic aromatic hydrocarbon. ${ }^{44,45}$ The depth of the $\mathrm{C}_{5 \mathrm{v}}$-symmetric bowl in corannulene, from the plane of the five-membered ring to the plane of rim carbon atoms, is $0.87 \AA .^{46}$ The planar structure of corannulene is a first-order saddle point on the PES connecting the two symmetry-equivalent bowl-shaped local minima. The energy barrier for the bowl-to-bowl inversion has been estimated from dynamic ${ }^{1} \mathrm{H}$ NMR measurements of ethyl- and bromomethyl-substituted corannulene derivatives to be $48.1 \mathrm{~kJ} \mathrm{~mol}^{-1} .^{47}$ Figure 2 gives a schematic representation of the uncatalyzed and catalyzed Gibbs free energy profiles. For the uncatalyzed reaction we obtain a barrier of $\Delta G^{\ddagger} 298$,uncat $=47.3 \mathrm{~kJ} \mathrm{~mol}^{-1}$, in excellent agreement with the experimental value. ${ }^{47}$ 


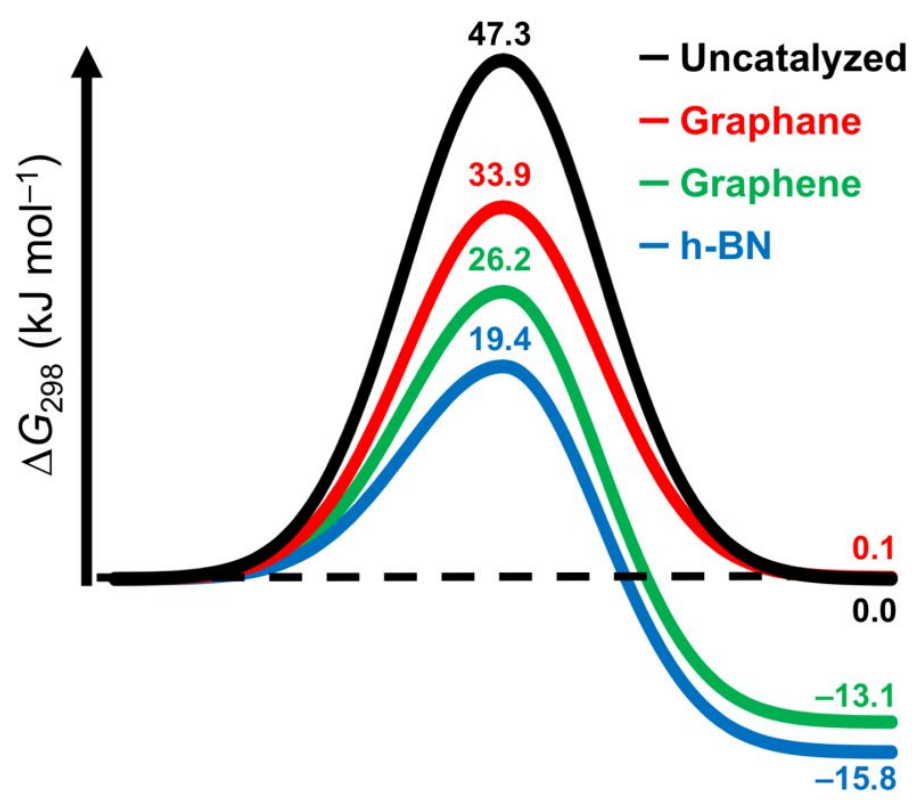

Figure 2. Gibbs free potential energy profiles $\left(\Delta G_{298}\right.$, revDSD-PBEP86-D3BJ/Def2-TZVPP, $\mathrm{kJ} \mathrm{mol}^{-1}$ ) for the bowl-to-bowl inversion in corannulene: uncatalyzed (black curve) and catalyzed by graphane (red curve), graphene (green curve), and h-BN (blue curve). The structures located along the catalyzed reaction profiles are shown in Figure 3.

Graphene catalysis. A graphene catalyst reduces the uncatalyzed barrier by $44.6 \%$ $\left(\Delta G^{\star} 298\right.$,graphene $\left.=26.2 \mathrm{~kJ} \mathrm{~mol}^{-1}\right)$. Taking the catalytic efficiency of the catalyst $\left(\Delta \Delta G_{298, \text { cat }}^{\star}\right)$ as the difference in barrier between the uncatalyzed $\left(\Delta G^{\dagger} 298\right.$,uncat $)$ and catalyzed $\left(\Delta G_{2}^{\dagger} 298\right.$,cat $)$ reaction barriers (i.e., $\Delta \Delta G^{\ddagger} 298$,graphene $=\Delta G^{\ddagger} 298$,uncat $-\Delta G^{\ddagger} 298$, graphene), we obtain $\Delta \Delta G^{\ddagger} 298$,graphene $=21.1 \mathrm{~kJ} \mathrm{~mol}^{-1}$. The catalytic efficiencies are listed in Table 1. According to the Eyring equation this catalytic enhancement corresponds to an increase of about four orders of magnitude in the reaction rate at $298 \mathrm{~K}$. The reactant complex $(\mathrm{RC})$, transition state structure (TSS), and product complex (PC) located along the catalyzed reaction profiles are shown in Figure 3. The PC in which the convex side of corannulene is facing the graphene catalyst is more stable by $13.1 \mathrm{~kJ} \mathrm{~mol}^{-1}$ than the $\mathrm{RC}$ in which the concave side of corannulene is facing graphene (see Figures 2 and 3). This greater stability of the PC is attributed to the larger $\pi$ - 
system on the convex side of corannulene leading to increased $\pi$-interactions with the graphene catalyst. ${ }^{48,49,50}$ Table S1 of the Supporting Information gives the revDSD-PBEP86D3BJ/Def2-TZVPP complexation energies between corannulene and the catalyst in the RC, TSS, and PC. We can estimate the interaction energy between corannulene and the catalyst in the TSS as the energy required to separate the catalyzed TSS into the free TSS and catalyst. This estimated TSS stabilization energy amounts to $195.2 \mathrm{~kJ} \mathrm{~mol}^{-1}$ and is greater than the RC and PC stabilization energies by 174.1 and $187.2 \mathrm{~kJ} \mathrm{~mol}^{-1}$, respectively. Thus, it is evident that shape complementarity between the planar TSS and graphene catalyst maximizes the overlap between the two $\pi$ systems which in turn results in greater stabilization of the TSS relative to the curved local minima. 


\section{a) Graphene catalyst}
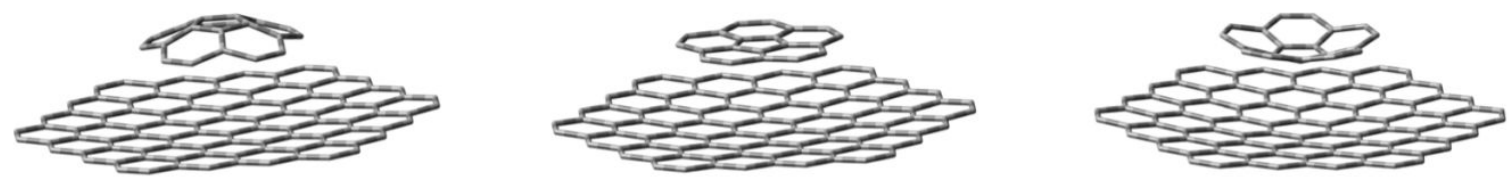

b) h-BN catalyst
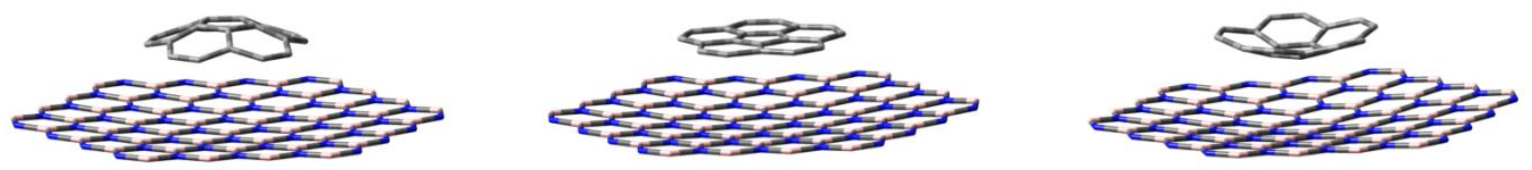

\section{c) Graphane catalyst}
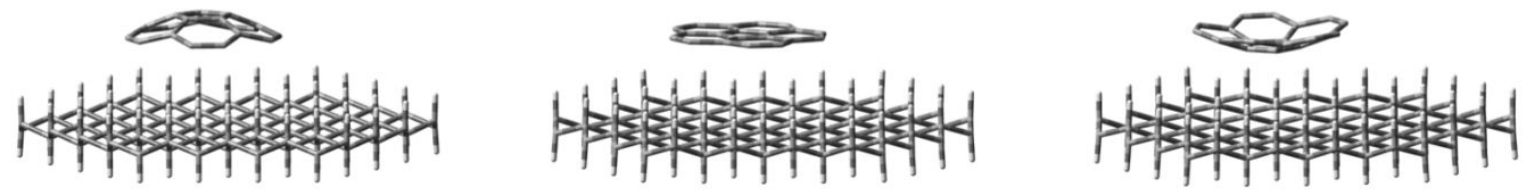

Figure 3. Optimized structures of the reactant complexes, transition state structures, and product complexes involved in the bowl-to-bowl inversion in corannulene catalyzed by (a) graphene, (b) h-BN, and (c) graphane. For clarity hydrogen atoms are omitted, with the exception of the axial hydrogens in graphane. Atomic color scheme: H, white; B, pink; C, gray; $\mathrm{N}$, blue. 
Table 1. Gibbs free activation energies at $298 \mathrm{~K}\left(\Delta G^{\star} 298\right)$ for the uncatalyzed and catalyzed processes and catalytic enhancements $\left(\Delta \Delta G^{\dagger} 298\right.$,cat $)$ for the graphene, h-BN, and graphane catalysts (revDSD-PBEP86-D3BJ/Def2-TZVPP, $\mathrm{kJ} \mathrm{mol}^{-1}$ ).

\begin{tabular}{llcc}
\hline & Catalyst & $\Delta G^{\ddagger}{ }_{298}$ & $\Delta \Delta G^{\dagger}{ }_{298, \text { cat }}{ }^{a}$ \\
\hline Corannulene bowl-to-bowl inversion & Uncatalyzed & 47.3 & \\
& Graphene & 26.2 & 21.1 \\
& h-BN & 19.4 & 27.9 \\
& Graphane & 33.9 & 13.4 \\
Sumanene bowl-to-bowl inversion & Uncatalyzed & 85.0 & \\
& Graphene & 39.2 & 45.8 \\
& h-BN & 56.6 & 28.4 \\
& Graphane & 72.2 & 12.8 \\
& Uncatalyzed & 83.7 & \\
& Graphene & 33.5 & 50.2 \\
& h-BN & 39.5 & 44.3 \\
& Graphane & 50.1 & 33.7 \\
\hline
\end{tabular}

${ }^{a} \Delta \Delta G_{298, \text { cat }}^{\ddagger}=\Delta G_{298, \text { uncat }}^{\ddagger}-\Delta G_{298, \text { cat }}^{\ddagger}$

h-BN catalysis. Remarkably, an h-BN catalyst results in a catalyzed Gibbs free energy barrier $\left(\Delta G^{*}{ }_{298, \mathrm{~h}-\mathrm{BN}}\right)$ of merely $19.4 \mathrm{~kJ} \mathrm{~mol}^{-1}$ relative to the reactant complex (Figure 2). This translates to a catalytic enhancement of $\Delta \Delta G^{\star} 298, \mathrm{~h}-\mathrm{BN}=27.9 \mathrm{~kJ} \mathrm{~mol}^{-1}$ for the h-BN catalyst (Table 1). We note that this catalytic enhancement corresponds to an increase of about five orders of magnitude in the reaction rate at $298 \mathrm{~K}$. Thus, h-BN is a more effective catalyst for the corannulene bowl-to-bowl inversion than graphene, for which we obtain a catalytic enhancement of $\Delta \Delta G_{298, \mathrm{~h}-\mathrm{BN}}^{\star}=21.1 \mathrm{~kJ} \mathrm{~mol}^{-1}$ (Table 1). Similar to the graphene-catalyzed corannulene inversion, the $\mathrm{PC}$ is more stable by $15.8 \mathrm{~kJ} \mathrm{~mol}^{-1}$ than the $\mathrm{RC}$. The greater stability of the PC is again attributed to the larger $\pi$-system on the convex side of corannulene. ${ }^{48,49,50}$

Inspection of Table S1 reveals that the interaction energy between h-BN and the corannulene reactant $\left(68.1 \mathrm{~kJ} \mathrm{~mol}^{-1}\right)$ is significantly smaller than that with graphene $(174.1 \mathrm{~kJ}$ $\mathrm{mol}^{-1}$ ). However, relative to these RC stabilization energies, the stabilization of the TSS is more pronounced by the h-BN catalyst (Table S1). In the PC, the interaction energy between 
corannulene and h-BN $\left(83.9 \mathrm{~kJ} \mathrm{~mol}^{-1}\right)$ is less than half of that between the corannulene product and graphene $\left(187.2 \mathrm{~kJ} \mathrm{~mol}^{-1}\right)$. The smaller interaction energy between corannulene and h-BN may be important in practical applications since it would ease the separation of the product from the heterogeneous catalyst.

Graphane catalysis. Let us now proceed to examine the corannulene bowl-to-bowl inversion catalyzed by a graphane catalyst. Inspection of Figure 2 reveals that graphane can catalyze the corannulene bowl-to-bowl inversion, albeit not as efficiently as graphene and h-BN. Nevertheless, a graphane catalyst still reduces the reaction barrier height by a chemically significant amount, i.e., from $\Delta G_{298 \text {,uncat }}^{\dagger}=47.3$ to $\Delta G^{\ddagger} 298$,graphane $=33.9 \mathrm{~kJ} \mathrm{~mol}^{-1}$. This reduction in the barrier height by $\Delta \Delta G_{298, \text { graphane }}^{\star}=13.4 \mathrm{~kJ} \mathrm{~mol}^{-1}$ corresponds to an increase in the reaction rate by about two orders of magnitude at $298 \mathrm{~K}$. In contrast to graphene and h$\mathrm{BN}$ where the PC is more stable than the RC due to the larger $\pi$-system of corannulene on the outside of the bowl, in graphane the RC and PC are essentially isoenergetic (Figure 2). The relatively small attractive $\sigma / \pi$-interactions between the graphane catalyst and corannulene in the $\mathrm{RC}$ and $\mathrm{PC} \sim 45.5 \mathrm{~kJ} \mathrm{~mol}^{-1}$ (Table S1) make graphane the most attractive catalyst from the perspective of product separation.

\section{Sumanene bowl-to-bowl inversion}

Uncatalyzed reaction. We turn now to the bowl-to-bowl inversion in sumanene. Figure 4 gives a schematic representation of the uncatalyzed and catalyzed Gibbs free energy profiles. For the uncatalyzed sumanene inversion we obtain a Gibbs free energy barrier of $\Delta G^{\dagger} 298$,uncat $=85.0 \mathrm{~kJ} \mathrm{~mol}^{-1}$. This reaction barrier height is practically spot on the experimental value obtained by Amaya et al. ${ }^{51}$ for trideuteriosumanene from 2D NMR measurements at $303 \mathrm{~K}$ $\left(\Delta G^{\dagger}{ }_{303 \text {,uncat }}=84.9 \mathrm{~kJ} \mathrm{~mol}^{-1}\right)$. 


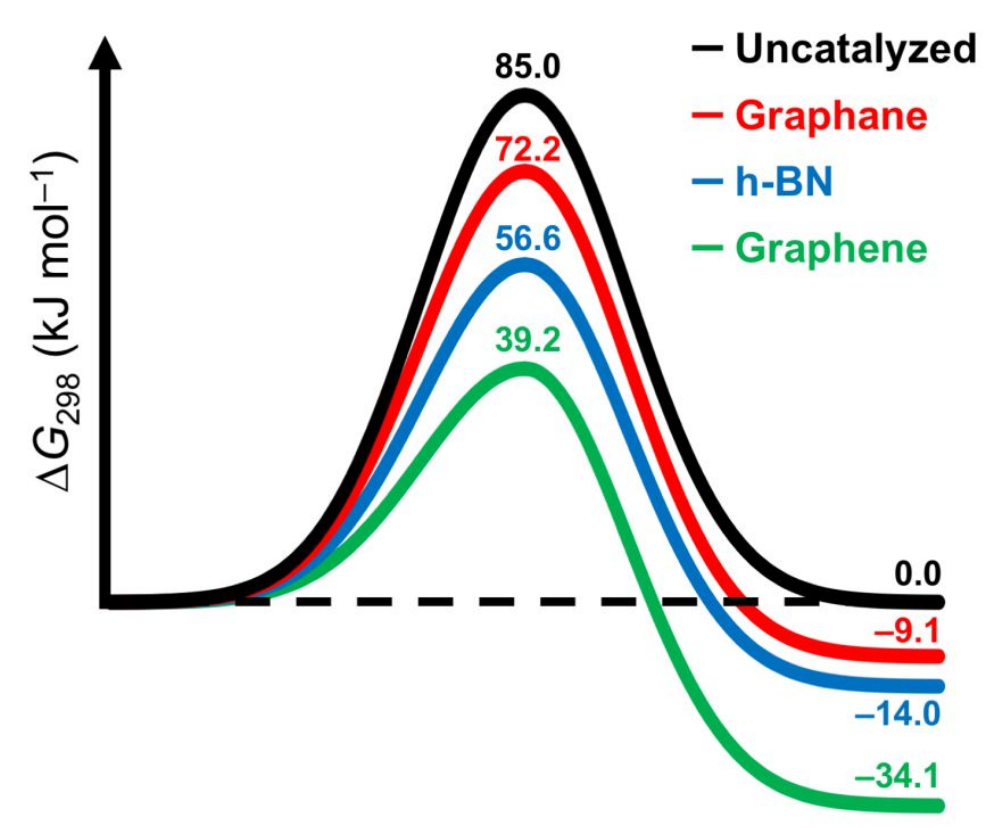

Figure 4. Gibbs free potential energy profiles $\left(\Delta G_{298}\right.$, revDSD-PBEP86-D3BJ/Def2-TZVPP, $\mathrm{kJ} \mathrm{mol}^{-1}$ ) for the bowl-to-bowl inversion in sumanene: uncatalyzed (black curve) and catalyzed by graphane (red curve), h-BN (blue curve), and graphene (green curve). The structures located along the catalyzed reaction profiles are shown in Figure 5.

Graphene catalysis. Figure 5 shows the RC, TSS, and PC located along the graphenecatalyzed reaction profile. A graphene catalyst results in a reaction barrier height of $\Delta G_{298, \text { graphene }}^{\ddagger}=39.2 \mathrm{~kJ} \mathrm{~mol}^{-1}$, i.e., it reduces the uncatalyzed reaction barrier by as much as $45.8 \mathrm{~kJ} \mathrm{~mol}^{-1}$. According to the Eyring equation, this catalytic enhancement corresponds to an increase of $\sim 8$ orders of magnitude in the reaction rate at $298 \mathrm{~K}$. Similar to the graphenecatalyzed bowl-to-bowl inversion in corannulene, this catalytic enhancement originates from the greater stabilization energy provided by the catalyst to the planar TSS than to the nonplanar RC. Specifically, the attractive $\pi$-interaction energies between the catalyst and substrate are estimated to be 136.3 (RC) and 182.1 (TSS) $\mathrm{kJ} \mathrm{mol}^{-1}$ (Table S1). We also note that consistent with the results for corannulene, the PC, in which the convex side of 
sumanene is pointing towards the graphene catalyst, is more stable than the RC by $34.1 \mathrm{~kJ}$ $\mathrm{mol}^{-1}$.

\section{a) Graphene catalyst}
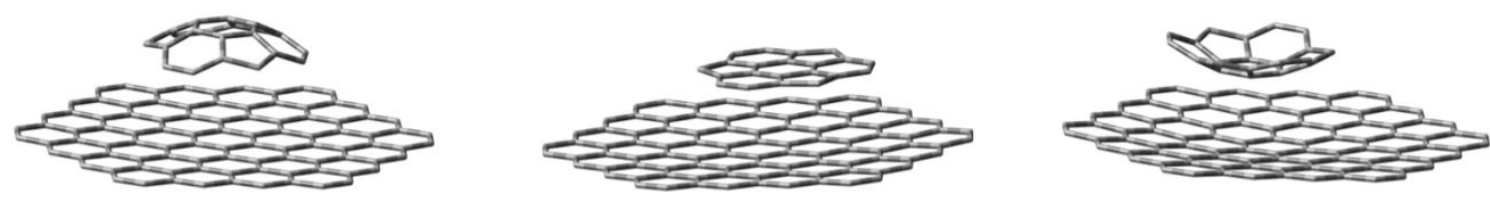

\section{b) h-BN catalyst}
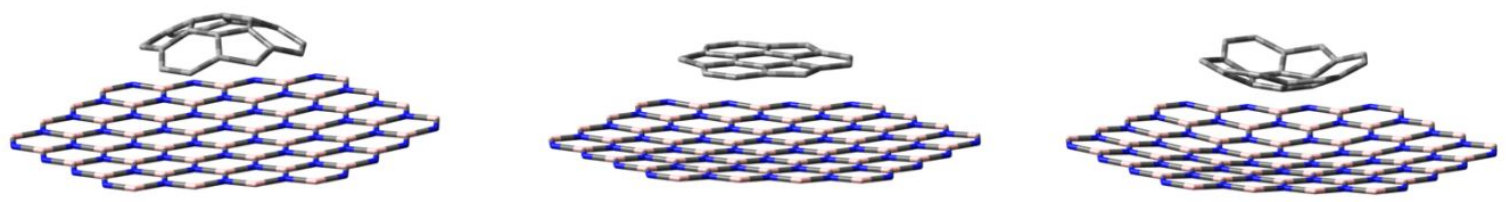

\section{c) Graphane catalyst}
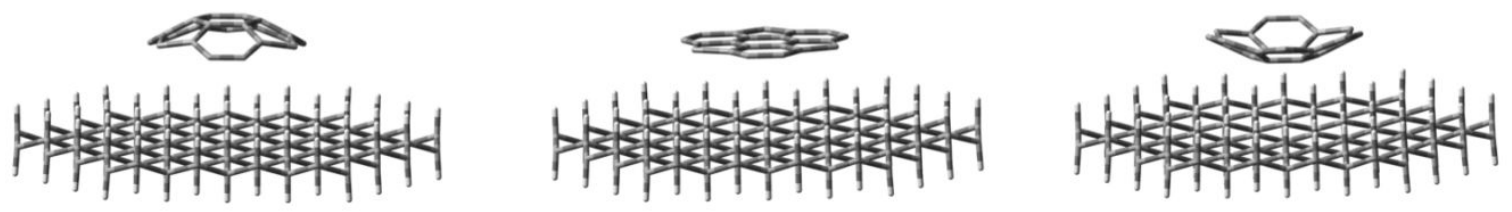

Figure 5. Optimized structures of the reactant complexes, transition state structures, and product complexes involved in the bowl-to-bowl inversion in sumanene catalyzed by (a) graphene, (b) h-BN, and (c) graphane. For clarity hydrogen atoms are omitted, with the exception of the axial hydrogens in graphane.

h-BN catalysis. The h-BN catalyst reduces the barrier height by an appreciable amount of $33.4 \%$ relative to the uncatalyzed barrier. In particular, we obtain $\Delta G^{\ddagger} 298, \mathrm{~h}-\mathrm{BN}=56.6 \mathrm{~kJ} \mathrm{~mol}^{-1}$, which leads to a catalytic efficiency of $\Delta \Delta G^{\dagger} 298, \mathrm{~h}-\mathrm{BN}=28.4 \mathrm{~kJ} \mathrm{~mol}^{-1}$ (Table 1). This barrier reduction translates to a rate enhancement of about five orders of magnitude relative to the uncatalyzed processes at $298 \mathrm{~K}$. The interaction energy between sumanene and h-BN amounts to $65.1 \mathrm{~kJ} \mathrm{~mol}^{-1}$ in the $\mathrm{RC}$ and $93.5 \mathrm{~kJ} \mathrm{~mol}^{-1}$ in the TSS (Table S1). Thus, again we 
see that shape complementarity between the planar catalyst and TSS results in a significant catalytic enhancement.

An interesting observation is that in the corannulene bowl-to-bowl inversion, h-BN $\left(\Delta \Delta G_{298, \mathrm{~h}-\mathrm{BN}}^{*}=27.9 \mathrm{~kJ} \mathrm{~mol}^{-1}\right.$, Table 1) shows a higher catalytic efficiency than graphene $\left(\Delta \Delta G_{298, \text { graphene }}^{\star}=21.1 \mathrm{~kJ} \mathrm{~mol}^{-1}\right)$. However, in the sumanene bowl-to-bowl inversion, graphene $\left(\Delta \Delta G_{298, \text { graphene }}^{\dot{1}}=45.8 \mathrm{~kJ} \mathrm{~mol}^{-1}\right)$ is a more efficient catalyst than h-BN $\left(\Delta \Delta G_{2}^{\dot{\dagger}} 298, \mathrm{~h}-\mathrm{BN}\right.$ $=28.4 \mathrm{~kJ} \mathrm{~mol}^{-1}$ ). These catalytic enhancements reveal that h-BN achieves similar catalytic efficiencies for both inversions, namely $\Delta \Delta G_{298, \mathrm{~h}-\mathrm{BN}}^{\ddagger}=27.9$ and $28.4 \mathrm{~kJ} \mathrm{~mol}^{-1}$ for the corannulene and sumanene bowl-to-bowl inversions, respectively. However, graphene catalyzes the sumanene bowl-to-bowl inversion more efficiently than the corannulene bowlto-bowl inversion. Namely, $\Delta \Delta G_{298 \text {,graphene }}^{\dagger}=21.1$ and $45.8 \mathrm{~kJ} \mathrm{~mol}^{-1}$ for the corannulene and sumanene inversions, respectively. This difference in catalytic efficiencies may be partially attributed to a better overlap between the planar triphenylene skeleton in the TSS and graphene catalyst for the sumanene inversion (for a top-down view of the TSSs, see Figure S1 of the Supporting Information).

Graphane catalysis. As is the case for the corannulene bowl-to-bowl inversion, graphane only has a modest catalytic efficiency, namely a graphane catalyst reduces the barrier height for the sumanene bowl-to-bowl inversion by $15.1 \%$ relative to the uncatalyzed barrier. Namely, the reaction barrier height is reduced from $\Delta G_{298 \text {,uncat }}^{\ddagger}=85.0$ to $\Delta G_{298 \text {, graphane }}^{\ddagger}=72.2$ $\mathrm{kJ} \mathrm{mol}^{-1}$. As is the case for corannulene, this reduction in the barrier height by $\Delta \Delta G^{\ddagger} 298$,graphane $=12.8 \mathrm{~kJ} \mathrm{~mol}^{-1}$ translates to an increase in the reaction rate by about two orders of magnitude at $298 \mathrm{~K}$.

It is also noteworthy that in contrast the graphene-catalyzed inversion of corannulene where the RC and PC are essentially isoenergetic, in the graphene-catalyzed inversion of 
sumanene the $\mathrm{PC}$ is more stable than the $\mathrm{RC}$ by $9.1 \mathrm{~kJ} \mathrm{~mol}^{-1}$. Inspection of the structures indicates that this stability is partially attributed to $\sigma-\pi$ interactions between the graphane's hydrogens and central $\mathrm{Ph}$ ring of sumannene, which is situated parallel to the graphane plane $\sim 2.44 \AA$ above the hydrogens. These $\sigma-\pi$ interactions are stronger in the PC where the concave side of sumanene is facing the graphane (Figure 5c).

\section{2,2'-Dimethylbiphenyl $\mathrm{C}-\mathrm{C}$ rotation}

Uncatalyzed reaction. To demonstrate the generality of catalysis by pristine $2 \mathrm{D}$ materials beyond bowl-to-bowl inversions, we consider as a final example the rotation around the $\mathrm{Ph}-$ $\mathrm{Ph}$ bond in 2,2'-dimethylbiphenyl. Biphenyl is a non-planar aromatic compound, in which competition between $\pi$-conjugation (which favors a coplanar structure) and steric repulsion of the ortho substituents (which favors a non-planar structure) result in a twist angle $(\alpha)$ between the two $\mathrm{Ph}$ rings. In 2,2'-dimethylbiphenyl the strong steric repulsion between the ortho methyl groups leads to the two phenyl rings being nearly orthogonal with $\alpha=92.7^{\circ}$. The conversion between the two axial enantiomers proceeds through a planar TSS with $\alpha=$ $0.0^{\circ}$. Our uncatalyzed rotational barrier $\left(\Delta G^{*} 298\right.$,uncat $=83.7 \mathrm{~kJ} \mathrm{~mol}^{-1}$, Figure 6$)$ is bracketed in between accurate $\operatorname{CCSD}(\mathrm{T}) / \mathrm{CBS}$ (i.e., coupled cluster with single, double, and quasiperturbative triple excitations at the complete basis set limit) estimations obtained via thermochemical protocols, ${ }^{52,53}$ namely: 86.5 (G4), ${ }^{54} 83.7 \quad(\mathrm{G} 4(\mathrm{MP} 2)),{ }^{55}$ and 82.0 $(\mathrm{CBS}-\mathrm{QB} 3)^{56} \mathrm{~kJ} \mathrm{~mol}^{-1}$. 


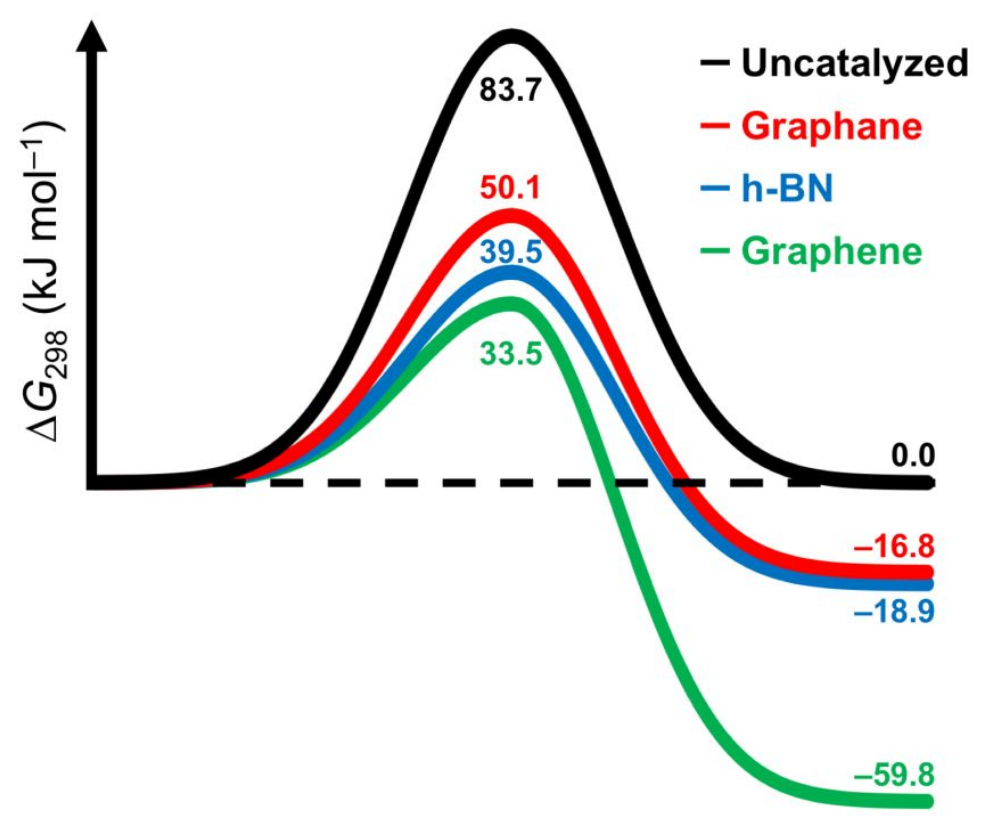

Figure 6. Gibbs free potential energy profiles $\left(\Delta G_{298}\right.$, revDSD-PBEP86-D3BJ/Def2-TZVPP, $\mathrm{kJ} \mathrm{mol}^{-1}$ ) for the rotation about the central $\mathrm{C}-\mathrm{C}$ bond in 2,2'-dimethylbiphenyl: uncatalyzed (black curve), catalyzed by graphane (red curve), catalyzed by h-BN (blue curve), and catalyzed by graphene (green curve). The structures located along the catalyzed reaction profiles are shown in Figure 7.

Graphene catalysis. The RCs, TSSs, and PCs located along the catalyzed reaction profiles are shown in Figure 7. In all the catalyzed processes (graphene, h-BN, and graphane), the methyl groups of $2,2^{\prime}$-dimethylbiphenyl in the RC are pointing towards the catalyst such that neither of the $\mathrm{Ph}$ rings is parallel to the $2 \mathrm{D}$ surface. In the $\mathrm{PC}$, however, one of the $\mathrm{Ph}$ rings lies parallel to the $2 \mathrm{D}$ surface, whilst the methyl group on the other ring points upwards. Thus, for all the considered $2 \mathrm{D}$ catalysts the $\mathrm{PC}$ is more stable than the $\mathrm{RC}$ due to the stronger $\pi-\pi$ and $\sigma-\pi$ interactions between the catalyst and the Ph ring lying parallel to the $2 \mathrm{D}$ catalyst (Figures 6 and 7). 


\section{a) Graphene catalyst}
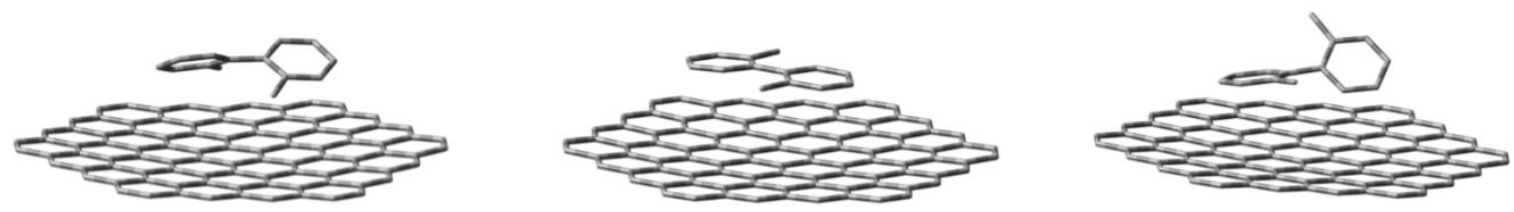

b) h-BN catalyst
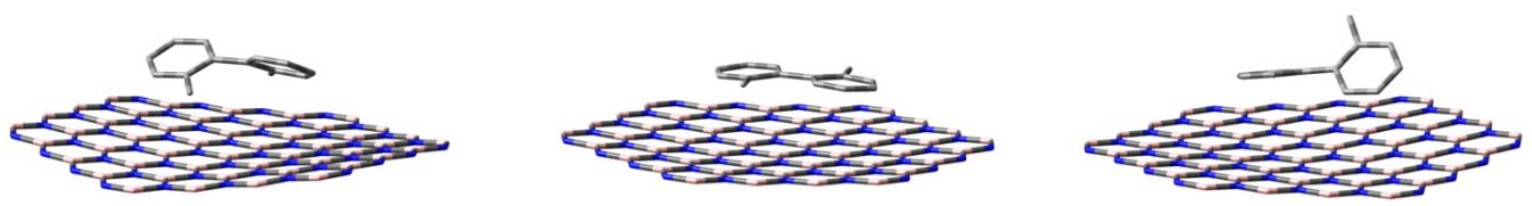

\section{c) Graphane catalyst}
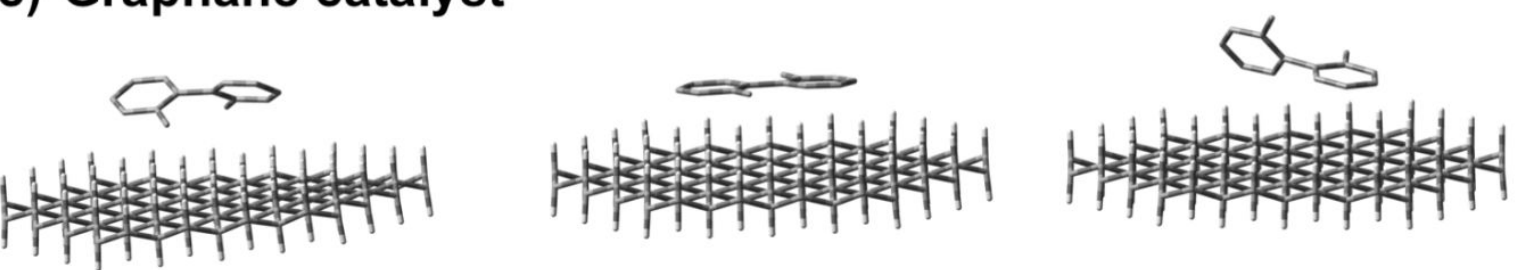

Figure 7. Optimized structures of the reactant complexes, transition state structures, and product complexes involved in the rotation about the central $\mathrm{C}-\mathrm{C}$ bond in 2,2'dimethylbiphenyl catalyzed by (a) graphene, (b) h-BN, and (c) graphane. For clarity hydrogen atoms are omitted, with the exception of the axial hydrogens in graphane.

A graphene catalyst reduces the uncatalyzed barrier height by a significant amount of $60.0 \%$. Namely, we obtain $\Delta G_{298 \text {,graphene }}^{\ddagger}=33.5 \mathrm{~kJ} \mathrm{~mol}^{-1}$ (Figure 6) and $\Delta \Delta G^{\ddagger}{ }_{298, \text { graphene }}=50.2$ $\mathrm{kJ} \mathrm{mol}{ }^{-1}$. Similar to the corannulene and sumanene inversions, the catalyst stabilizes the planar TSS $\left(142.7 \mathrm{~kJ} \mathrm{~mol}^{-1}\right)$ to a greater extent than the non-planar reactant $\left(92.5 \mathrm{~kJ} \mathrm{~mol}^{-1}\right.$, Table S1). It is noteworthy that the strong $\pi$-interactions between the graphene catalyst and 2,2'-dimethylbiphenyl 'flattens' the 2,2'-dimethylbiphenyl in both the RC and PC. In particular, we obtain dihedral angles of $\alpha=45.5^{\circ}(\mathrm{RC})$ and $\alpha=51.4^{\circ}$ (PC) (cf. $\alpha=92.7^{\circ}$ in free 2,2'-dimethylbiphenyl). Thus, in addition TSS stabilization due to shape complementarity between catalyst and TSS, the graphene catalyst destabilizes the reactant 
and product by forcing 2,2'-dimethylbiphenyl to adopt a flatter conformation, which contributes to the reduction in the energy barrier for the rotation process.

h-BN catalysis. An h-BN catalyst reduces the uncatalyzed reaction barrier for the rotation around the central $\mathrm{C}-\mathrm{C}$ bond by $52.8 \%$. Namely, we obtain $\Delta G^{\dagger} 298, \mathrm{~h}-\mathrm{BN}=39.5 \mathrm{~kJ} \mathrm{~mol}^{-1}$ and $\Delta \Delta G_{298, \mathrm{~h}-\mathrm{BN}}=44.2 \mathrm{~kJ} \mathrm{~mol}^{-1}$ (Figure 6). Thus, an h-BN catalyst has a similar catalytic efficiency to that of graphene $\left(\Delta \Delta G^{\ddagger}{ }_{298 \text {,graphene }}=50.2 \mathrm{~kJ} \mathrm{~mol}{ }^{-1}\right)$, but with the important advantage that it binds the reaction product less strongly than graphene, namely with a binding Gibbs free energy of $31.5 \mathrm{~kJ} \mathrm{~mol}^{-1}$ (Table S1). Thus, h-BN is a more suitable catalyst from the perspective of product separation. As expected the catalytic effect originates from shape complementarity between catalyst and TSS, namely the h-BN catalyst stabilizes the species located along the reaction coordinate by 12.7 (RC), 56.9 (TSS), and 31.5 (PC) kJ $\mathrm{mol}^{-1}$ (Table S1).

Graphane catalysis. As is the case for the two bowl-to-bowl inversions, graphane catalyzes the rotation about the $\mathrm{C}-\mathrm{C}$ bond in 2,2'-dimethylbiphenyl to a lesser extent than graphene and h-BN. Nevertheless, graphane still catalyzes this rotation to a remarkable degree. Namely, a graphane catalyst reduces the reaction barrier from $\Delta G_{298 \text {,uncat }}^{\star}=83.7$ to merely $\Delta G_{298, \text { graphane }}^{\ddagger}=50.1 \mathrm{~kJ} \mathrm{~mol}^{-1}$. This reduction in the barrier height by $\Delta \Delta G_{298, \text { graphane }}^{\dagger}=40.1 \mathrm{~kJ}$ $\mathrm{mol}^{-1}$ corresponds to an increase in the reaction rate by about six orders of magnitude at 298 K. Moreover, graphane binds the product with a Gibbs free binding energy of merely $8.7 \mathrm{~kJ}$ $\mathrm{mol}^{-1}$, making it an attractive catalyst from the perspective of product separation. 
Nature of noncovalent interactions responsible for the catalytic activity of pristine 2D materials

As discussed above, and illustrated in Figures 3, 5, and 7 the planar geometry of the inversion and rotation TSSs maximizes the noncovalent interactions with the 2D catalysts relative to the non-planar reactants and products. In order to shed light on the energy components of the noncovalent interactions responsible for these catalytic effects, we carried out an energy decomposition analysis using the second generation ALMO-EDA scheme by Head-Gordon and co-workers. ${ }^{39,40}$ This analysis gives the component breakdown of the intermolecular interaction energy into dispersion $\left(\Delta E_{\text {disp }}\right)$, electrostatic $\left(\Delta E_{\text {elec }}\right)$, Pauli repulsion $\left(\Delta E_{\text {pauli }}\right)$, polarization $\left(\Delta E_{\mathrm{pol}}\right)$, and charge transfer $\left(\Delta E_{\mathrm{ct}}\right)$ interactions. Table $\mathrm{S} 2$ of the Supporting Information lists the contributions of these components $\left(\Delta \Delta E_{\text {comp }}\right)$ to the reaction barrier heights of the catalyzed reactions, i.e., $\Delta \Delta E_{\text {comp }}=\Delta E_{\text {comp }}(\mathrm{TSS})-\Delta E_{\text {comp }}(\mathrm{RC})$. A negative $\Delta \Delta E_{\text {comp }}$ value indicates stabilization of the TSS relative to the RC, whilst a positive value indicates a repulsive interaction. The results in Table S2 show that the polarization and charge transfer terms are very small and normally amount to less than $1 \mathrm{~kJ}$ $\mathrm{mol}^{-1}$. The only exceptions to this are the processes catalyzed by h-BN, for which the charge transfer term can reach up to $4.7 \mathrm{~kJ} \mathrm{~mol}^{-1}$.

The electrostatic and dispersion contributions to the catalyzed reaction barrier heights are depicted in Figure 8. As expected, the electrostatic $\left(\Delta \Delta E_{\text {elec }}\right)$ and dispersion $\left(\Delta \Delta E_{\text {disp }}\right)$ interactions systematically stabilize the TSSs relative to the RCs by significant amounts, whilst the Pauli term ( $\left.\Delta \Delta E_{\text {pauli }}\right)$ destabilizes the TSSs. In all cases both the electrostatic and dispersion contributions are significantly stronger in the more closely interacting transition state structure complexes compared to the less closely bound equilibrium structure complexes. In particular, dispersion interactions stabilize the TSSs by amounts ranging 
between $\Delta \Delta E_{\text {disp }}=28.3-59.6 \mathrm{~kJ} \mathrm{~mol}^{-1}$, and electrostatic interactions stabilize the TSSs by amounts ranging between $\Delta \Delta E_{\text {elec }}=19.7-55.6 \mathrm{~kJ} \mathrm{~mol}^{-1}$ (Figure 8). The Pauli repulsion, on the other hand, destabilizes the TSSs by amounts ranging between $\Delta \Delta E_{\text {pauli }}=26.4-81.5 \mathrm{~kJ}$ $\mathrm{mol}^{-1}$. However, it is important to note that in all cases the large stabilizing electrostatic and dispersion interactions outweigh the destabilizing Pauli repulsion.

In the h-BN catalyzed processes both the electrostatic and dispersion components are nearly equal to each other (Figure 8). However, in the graphene- and graphane-catalyzed processes, the dispersion stabilizations are somewhat more pronounced than the electrostatic stabilizations (Figure 8). These results are consistent with relatively large contributions from both electrostatic and dispersion interactions in large $\pi-\pi$ and $\sigma-\pi$ stacked hydrocarbons..$^{57,58,59,60}$ It should be emphasized, however, that in contrast to previous works which examined absolute interaction energies in stacked dimers, here we are mainly interested in the difference in these stabilizing interactions between the TSS and RC. The above EDA analysis shows that both dispersion and electrostatic interactions stabilize the TSSs to greater extents than the RCs due to shape complementarity between TSS and catalyst. 


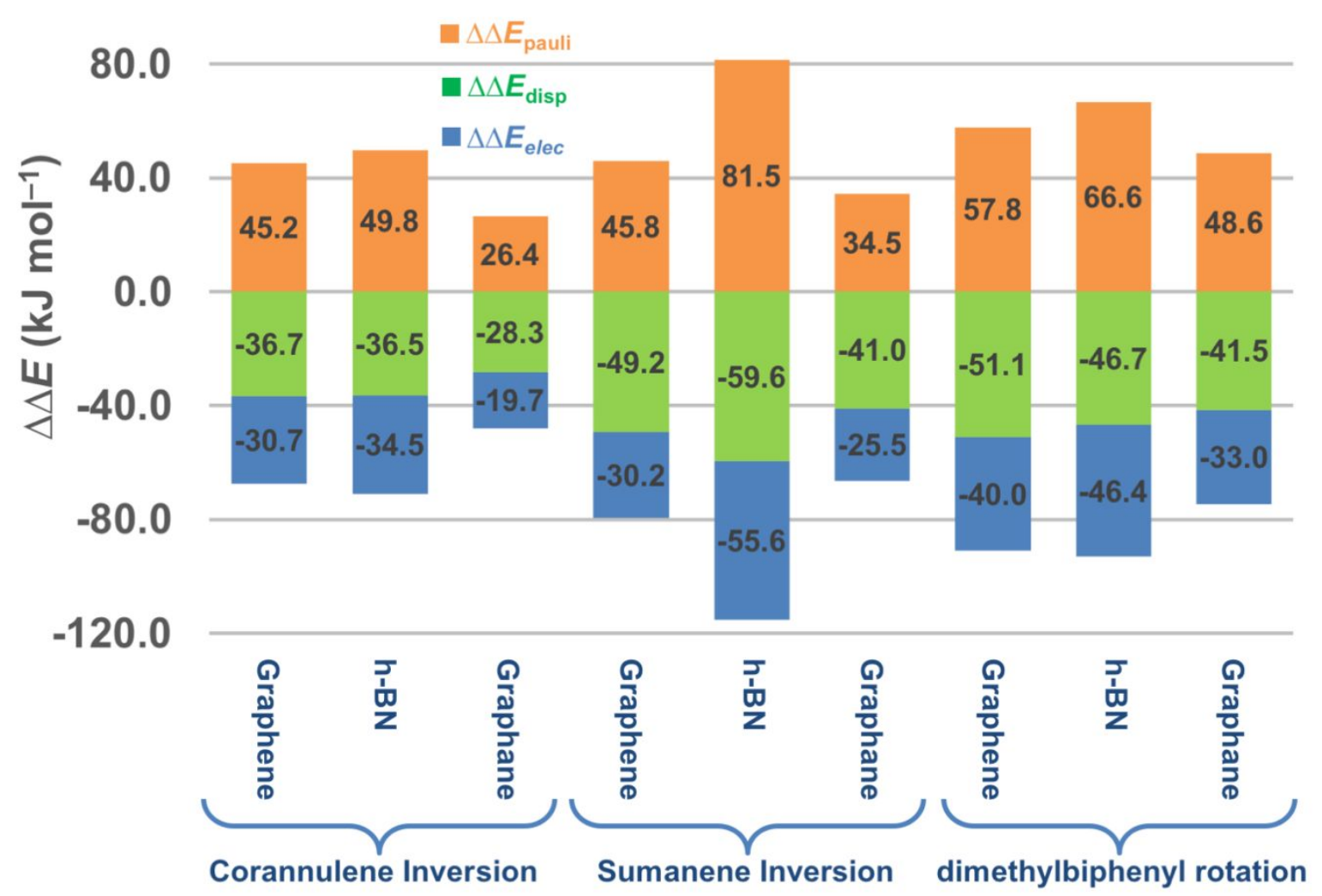

Figure 8. Component breakdown of the electrostatic $\left(\Delta \Delta E_{\text {elec }}\right)$, dispersion $\left(\Delta \Delta E_{\text {disp }}\right)$, and Pauli repulsion ( $\left.\Delta \Delta E_{\text {pauli }}\right)$ contributions to the catalyzed reaction barrier heights obtained from second-generation ALMO-EDA at the $\omega$ B97M-V/Def2-SVP level of theory. A negative $\Delta \Delta E$ value indicates stabilization (and a positive $\Delta \Delta E$ value indicates destabilization) of the TSS relative to the RC, see text and Table S2 of the Supporting Information.

\section{Conclusions}

It has been previously predicted computationally that pristine graphene can efficiently catalyze chemical processes which proceed via planar (or nearly planar) transition state structures. ${ }^{19,20,21}$ These predictions have been recently confirmed experimentally via chiral HPLC measurements, which showed that graphene can efficiently catalyze the racemization of 1,1'-binaphthyl-2,2'-diol (BINOL) via rotation about the $\mathrm{C}-\mathrm{C}$ bond connecting the two naphthyl units. ${ }^{22}$ Here we extend the scope of catalysis by pristine $2 \mathrm{D}$ materials by showing 
that other 2D nanomaterials can efficiently catalyze such chemical processes. In particular, we focus on prototypical 2D materials with different electronic structures and chemical compositions than graphene, namely: hexagonal boron nitride and graphane.

We show that both h-BN and graphane can catalyze the bowl-to-bowl inversion of corannulene and sumanene and the rotation about the $\mathrm{C}-\mathrm{C}$ bond in substituted biphenyls. $\mathrm{A}$ common feature shared by these catalytic processes is that they are all driven by shape complementarity between the planar 2D catalyst and planar transition state structure. Using high-level double-hybrid DFT calculations, we show that noncovalent $\pi$-interactions (or $\sigma / \pi$ interactions in the case of graphane) stabilize the planar TSS to a greater extent than they stabilize the non-planar local minima.

We find that h-BN catalyzes the bowl-to-bowl inversion in corannulene more efficiently than graphene. In particular, it reduces the uncatalyzed reaction barrier height $\left(\Delta G_{298 \text {,uncat }}^{*}=\right.$ $47.3 \mathrm{~kJ} \mathrm{~mol}^{-1}$ ) by $59 \%$ to $\Delta G^{\ddagger} 298, \mathrm{~h}-\mathrm{BN}=19.4 \mathrm{~kJ} \mathrm{~mol}^{-1}$, resulting in a catalytic enhancement of $\Delta \Delta G_{298, \mathrm{~h}-\mathrm{BN}}^{\star}=27.9 \mathrm{~kJ} \mathrm{~mol}^{-1}$. For comparison, graphene achieves a catalytic enhancement of $\Delta \Delta G_{298 \text {,graphene }}^{\ddagger}=21.1 \mathrm{~kJ} \mathrm{~mol}^{-1}$. On the other hand, the bowl-to-bowl inversion in sumanene is catalyzed more efficiently by graphene with $\Delta \Delta G^{\ddagger} 298$,graphene $=45.8 \mathrm{~kJ} \mathrm{~mol}^{-1}$ compared to a catalytic enhancement of $\Delta \Delta G^{\star} 298, \mathrm{~h}-\mathrm{BN}=28.4 \mathrm{~kJ} \mathrm{~mol}^{-1}$ for h-BN. For the rotation about the central $\mathrm{C}-\mathrm{C}$ bond in 2,2'-dimethylbiphenyl both graphene and h-BN result in large catalytic enhancements, namely of $\Delta \Delta G_{298, \text { graphene }}^{\ddagger}=50.2 \mathrm{~kJ} \mathrm{~mol}^{-1}$ and $\Delta \Delta G_{298, \mathrm{~h}-\mathrm{BN}}^{\ddagger}=44.2 \mathrm{~mol}^{-1}$.

Graphane is able to catalyze all the considered reactions, albeit to a lesser extent than graphene and h-BN. Nevertheless, graphane still achieves significant catalytic enhancements of $\Delta \Delta G_{298 \text {,graphane }}^{\ddagger}=13.4$ (corannulene bowl-to-bowl inversion), 12.8 (sumanene bowl-tobowl inversion), and 33.6 (C-C rotation in 2,2'-dimethylbiphenyl) $\mathrm{kJ} \mathrm{mol}^{-1}$. From a perspective of product separation, however, graphane has an advantage over both graphene 
and h-BN since it involves significantly smaller interaction energies with the reaction product.

Finally, we use a DFT-based energy decomposition analysis to reveal the nature of the intermolecular interactions responsible for these catalytic activities. We find that in all cases (graphene, h-BN, and graphane) catalysis is driven by both dispersion and electrostatic interactions, which together outweigh the Pauli repulsion term.

\section{Corresponding Author}

^A. Karton, E-Mail: amir.karton@uwa.edu.au

\section{Supporting Information}

Top-down view of corannulene and sumanene bowl-to-bowl inversions on graphene (Figure S1); ALMO-EDA analysis (Table S1); optimized geometries (Cartesian coordinates) for all species considered in the present work (Table S2); and full references for the Gaussian 16, ORCA 4.2.0, and Q-Chem 5.2 program suites.

\section{Acknowledgments}

We acknowledge resources from the National Computational Infrastructure (NCI), supported by the Australian Government, and system administration support provided by the Faculty of Science at the University of Western Australia to the Linux cluster of the Karton group. We gratefully acknowledge an Australian Research Council (ARC) Future Fellowship (Project No. FT170100373). 


\section{References}

${ }^{1}$ Hu, M.; Yao, Z.; Wang, X. Graphene-Based Nanomaterials for Catalysis. Ind. Eng. Chem. Res. 2017, 56, 3477-3502.

${ }^{2}$ Deng, D.; Fu Q.; Bao X.; Novoselov, K. S.; Zheng, N.; Tian Z. Catalysis with twodimensional materials and their heterostructures. Nat. Nanotechnol. 2016, 11, 218-230.

${ }^{3}$ Fan, X.; Zhang, G.; Zhang, F. Multiple roles of graphene in heterogeneous catalysis. Chem. Soc. Rev. 2015, 44, 3023-3035.

${ }^{4}$ Liu, M. M.; Zhang, R. Z.; Chen, W. Graphene-Supported Nanoelectrocatalysts for Fuel Cells: Synthesis, Properties, and Applications. Chem. Rev. 2014, 114, 5117-5160.

${ }^{5}$ Navalon, S.; Dhakshinamoorthy, A.; Alvaro, M.; Garcia, H. Carbocatalysis by GrapheneBased Materials. Chem. Rev. 2014, 114, 6179-6212.

${ }^{6}$ Su, D. S.; Perathoner, S.; Centi, G. Nanocarbons for the Development of Advanced Catalysts. Chem. Rev. 2013, 113, 5782-5816.

${ }^{7}$ Wang, H.; Dai, H. Strongly coupled inorganic-nano-carbon hybrid materials for energy storage. Chem. Soc. Rev. 2013, 42, 3088-3113.

${ }^{8}$ Brownson, D. A. C.; Kampouris, D. K.; Banks, C. E. Graphene electrochemistry: fundamental concepts through to prominent applications. Chem. Soc. Rev. 2012, 41, 69446976.

${ }^{9}$ Xiang, Q.; Yu, J.; Jaroniec, M. Graphene-based semiconductor photocatalysts. Chem. Soc. Rev. 2012, 41, 782-796.

${ }^{10}$ Chen, D.; Tang, L. H.; Li, J. H. Graphene-based materials in electrochemistry. Chem. Soc. Rev. 2010, 39, 3157-3180.

${ }^{11}$ Machado, B. F.; Serp, P. Graphene-based materials for catalysis. Catal. Sci. Technol. 2012, $2,54-75$. 
${ }^{12}$ Hobza, P.; Müller-Dethlefs K. Noncovalent Interactions. Theory and Experiment, first edition; Cambridge: The Royal Society of Chemistry, 2009.

${ }^{13}$ Schneider, H. J. Binding Mechanisms in Supramolecular Complexes. Angew. Chem. Int. Ed. 2009, 48, 3924-3977.

14 Gobre, V. V.; Tkatchenko, A. Scaling laws for van der Waals interactions in nanostructured materials. Nat. Commun. 2013, 4, 2341.

${ }^{15}$ Wagner, J. P.; Schreiner, P. R. London Dispersion in Molecular Chemistry-Reconsidering Steric Effects. Angew. Chem. Int. Ed. 2015, 54, 12274-12296.

${ }^{16}$ Su, G.; Yang, S.; Jiang, Y.; Li, J.; Li, S.; -C. Ren, J.; Liu W. Modeling chemical reactions on surfaces: the roles of chemical bonding and van der Waals interactions Prog. Surf. Sci. 2019, 94, 100561.

${ }^{17}$ Wheeler, S. E.; Seguin, T. J.; Guan, Y.; Doney, A. C. Noncovalent Interactions in Organocatalysis and the Prospect of Computational Catalyst Design. Acc. Chem. Res. 2016, 49, 1061-1069.

18 Toste, F. D.; Sigman, M. S.; Miller, S. J. Pursuit of Noncovalent Interactions for Strategic Site-Selective Catalysis. Acc. Chem. Res. 2017, 50, 609-615.

${ }^{19}$ Karton, A. Inversion and rotation processes involving non-planar aromatic compounds catalyzed by extended polycyclic aromatic hydrocarbons. Chem. Phys. Lett. 2014, 614, 156161.

${ }^{20}$ Denis, P. A. Pristine Graphene-Based Catalysis: Significant Reduction of the Inversion Barriers of Adsorbed and Confined Corannulene, Sumanene, and Dibenzo[a,g]corannulene. J. Phys. Chem. A 2015, 119, 5770-5777.

${ }^{21}$ Kroeger, A. A.; Karton, A. Catalysis by Pure Graphene-From Supporting Actor to Protagonist through Shape Complementarity. J. Org. Chem. 2019, 84, 11343-11347. 
${ }^{22}$ Kroeger, A. A.; Hooper, J. F.; Karton, A. Pristine graphene as a racemization catalyst for axially chiral BINOL. PhysChemPhys 2020, in press, DOI: 10.1002/cphc.202000426.

${ }^{23}$ Juríček, M.; Strutt, N. L.; Barnes, J. C.; Butterfield, A. M.; Dale, E. J.; Baldridge, K. K.; Stoddart, J. F.; Siegel, J. S. Induced-fit Catalysis of Corannulene Bowl-to-Bowl Inversion. Nat. Chem. 2014, 6, 222-228.

${ }^{24}$ Jaafar, R.; Pignedoli, C. A.; Bussi, G.; Aït-Mansour, K.; Groening, O.; Amaya, T.; Hirao, T.; Fasel, R.; Ruffieux, P. Bowl Inversion of Surface-Adsorbed Sumanene. J. Am. Chem. Soc. 2014, 136, 13666-13671.

${ }^{25}$ Wang, J.; Ma, F.; Sun, M. Graphene, hexagonal boron nitride, and their heterostructures: properties and applications. $R S C A d v$. 2017, 7, 16801-16822.

${ }^{26}$ Perdew, J. P.; Burke, K.; Ernzerhof, M. Generalized Gradient Approximation Made Simple. Phys. Rev. Lett. 1996, 77, 3865.

${ }^{27}$ Raghavachari, K.; Binkley, J. S.; Seeger, R.; Pople, J. A. Self-consistent molecular orbital methods. XX. A basis set for correlated wave functions. J. Chem. Phys. 1980, 72, 650.

${ }^{28}$ Grimme, S. Density functional theory with London dispersion corrections. WIREs Comput. Mol. Sci. 2011, 1, 211-228.

${ }^{29}$ Becke, A. D.; Johnson, E. R. A density-functional model of the dispersion interaction. $J$. Chem. Phys. 2005, 123, 154101.

${ }^{30}$ Grimme, S.; Ehrlich, S.; Goerigk, L. Effect of the damping function in dispersion corrected density functional theory. J. Comput. Chem. 2011, 32, 1456-1465.

${ }^{31}$ Gonzalez, C.; Schlegel, H. B. An improved algorithm for reaction path following. J. Chem. Phys. 1989, 90, 2154.

${ }^{32}$ Gaussian 16, Revision A.01, M. J. Frisch, et al. Gaussian, Inc., Wallingford CT, 2016. See also: http://www.gaussian.com. 
${ }^{33}$ Goerigk, L.; Grimme, S. Double-hybrid density functionals. WIREs Comput. Mol. Sci. 2014, 4, 576-600.

${ }^{34}$ Santra, G.; Sylvetsky, N.; Martin, J. M. L. Minimally Empirical Double-Hybrid Functionals Trained against the GMTKN55 Database: revDSD-PBEP86-D4, revDOD-PBED4, and DOD-SCAN-D4. J. Phys. Chem. A 2019, 123, 5129-5143.

${ }^{35}$ Martin, J. M. L.; Santra, G. Empirical Double-Hybrid Density Functional Theory: A 'Third Way' in Between WFT and DFT. Isr. J. Chem. 2020, in press, DOI: 10.1002/ijch.201900114. ${ }^{36}$ Goerigk, L.; Hansen, A.; Bauer, C.; Ehrlich, S.; Najibi, A.; Grimme, S. A look at the density functional theory zoo with the advanced GMTKN55 database for general main group thermochemistry, kinetics and noncovalent interactions. Phys. Chem. Chem. Phys. 2017, 19, 32184-32215.

${ }^{37}$ Weigend, F.; Ahlrichs, R. Balanced basis sets of split valence, triple zeta valence and quadruple zeta valence quality for H to Rn: Design and assessment of accuracy. Phys. Chem. Chem. Phys. 2005, 7, 3297-3305.

${ }^{38}$ Neese, F. The ORCA program system. WIREs Comput. Mol. Sci. 2011, 2, 73-78.

${ }^{39}$ Horn, P. R.; Mao, Y.; Head-Gordon, M. Probing non-covalent interactions with a second generation energy decomposition analysis using absolutely localized molecular orbitals. Phys. Chem. Chem. Phys. 2016, 18, 23067-23079.

${ }^{40}$ Mao, Y.; Horn, P. R.; Head-Gordon, M. Energy decomposition analysis in an adiabatic picture. Phys. Chem. Chem. Phys. 2017, 19, 5944-5958.

${ }^{41}$ Zhao, L.; von Hopffgarten, M.; Andrada, D. M.; Frenking, G. Energy decomposition analysis. WIREs Comput. Mol. Sci. 2018, 8, e1345. 
${ }^{42}$ Mardirossian, N.; Head-Gordon, M. $\omega$ B97M-V: A combinatorially optimized, rangeseparated hybrid, meta-GGA density functional with VV10 nonlocal correlation. J. Chem. Phys. 2016, 144, 214110.

${ }^{43}$ Shao, Y. et al. Advances in molecular quantum chemistry contained in the Q-Chem 4 program package. Mol. Phys. 2015, 113, 184-215.

${ }^{44}$ Wu, Y.-T.; Siegel, J. S. Aromatic Molecular-Bowl Hydrocarbons: Synthetic Derivatives, Their Structures, and Physical Properties. Chem. Rev. 2006, 106, 4843-4867.

${ }^{45}$ Tsefrikas, V. M.; Scott, L. T. Geodesic Polyarenes by Flash Vacuum Pyrolysis. Chem. Rev. 2006, 106, 4868-4884.

${ }^{46}$ Hanson, J. C.; Nordman, C. E. The crystal and molecular structure of corannulene, $\mathrm{C}_{20} \mathrm{H}_{10}$. Acta Crystallogr. B 1976, B32, 1147-1153.

${ }^{47}$ Seiders, T. J.; Baldridge, K. K.; Grube, G. H.; Siegel, J. S. Structure/Energy Correlation of Bowl Depth and Inversion Barrier in Corannulene Derivatives: Combined Experimental and Quantum Mechanical Analysis. J. Am. Chem. Soc. 2001, 123, 517-525.

${ }^{48}$ Grimme, S.; Antony, J.; Schwabe, T.; Mück-Lichtenfeld, C. Density functional theory with dispersion corrections for supramolecular structures, aggregates, and complexes of (bio)organic molecules. Org. Biomol. Chem. 2007, 5, 741-758.

${ }^{49}$ Martin, J. W.; Slavchov, R. I.; Yapp, E. K. Y.; Akroyd, J.; Mosbach, S.; Kraft, M. The Polarization of Polycyclic Aromatic Hydrocarbons Curved by Pentagon Incorporation: The Role of the Flexoelectric Dipole. J. Phys. Chem. C 2017, 121, 27154-27163.

${ }^{50}$ Cabaleiro-Lago, E. M.; Fernández, B.; Rodríguez-Otero, J. Dissecting the ConcaveConvex $\pi-\pi$ Interaction in Corannulene and Sumanene Dimers: SAPT(DFT) Analysis and Performance of DFT Dispersion-Corrected Methods. J. Comput. Chem. 2018, 39, 93-104. 
${ }^{51}$ Amaya, T.; Sakane, H.; Muneishi, T.; Hirao, T. Bowl-to-bowl inversion of sumanene derivatives. Chem. Commun. 2008, 765-767.

${ }^{52}$ Curtiss, L. A.; Redfern, P. C.; Raghavachari, K. Gn theory. WIREs Comput. Mol. Sci. 2011, $1,810-825$.

${ }^{53}$ Karton, A. A computational chemist's guide to accurate thermochemistry for organic molecules. WIREs Comput. Mol. Sci. 2016, 6, 292-310.

${ }^{54}$ Curtiss, L. A.; Redfern, P. C.; Raghavachari, K. Gaussian-4 theory. J. Chem. Phys. 2007, 126,084108

${ }^{55}$ Curtiss, L. A.; Redfern, P. C.; Raghavachari, K. Gaussian-4 theory using reduced order perturbation theory. J. Chem. Phys. 2007, 127, 124105.

${ }^{56}$ Montgomery, J. A.; Frisch, M. J.; Ochterski, J. W.; Petersson, G. A. A complete basis set model chemistry. VI. Use of density functional geometries and frequencies. J. Chem. Phys. 1999, 110, 2822.

57 Grimme, S. Do Special Noncovalent $\pi-\pi$ Stacking Interactions Really Exist?. Angew. Chem. Int. Ed. 2008, 47, 3430-3434.

${ }^{58}$ Ryno, S. M.; Risko, C.; Brédas, J.-L. Noncovalent Interactions and Impact of Charge Penetration Effects in Linear Oligoacene Dimers and Single Crystals. Chem. Mater. 2016, 28, $3990-4000$.

${ }^{59}$ Gryn'ova, G.; Corminboeuf, C. Steric “attraction”: not by dispersion alone. Beilstein J. Org. Chem. 2018, 14, 1482-1490.

${ }^{60}$ Cabaleiro-Lago, E. M.; Rodríguez-Otero, J. ACS Omega 2018, 3, 9348-9359. 
TOC Graphic

\section{Catalysis on pristine 2D materials via $\sigma / \pi$-interactions}

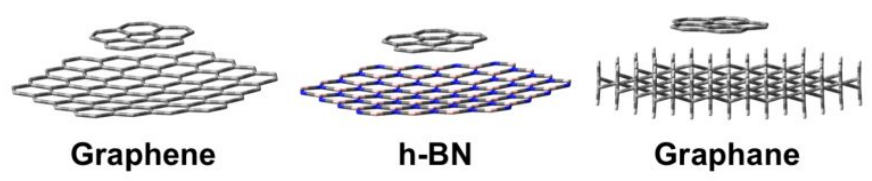




\section{Author biography}

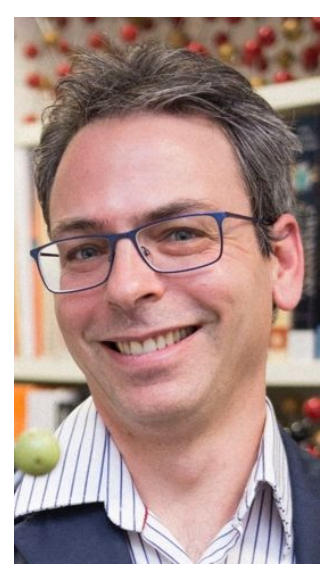

Associate Professor Amir Karton leads the computational chemistry group at the University of Western Australia. He obtained his PhD in computational chemistry in 2010 with Prof. Jan Martin at the Weizmann Institute of Science. During 2010-2, he was a postdoctoral fellow with Prof. Leo Radom at the University of Sydney. Amir currently holds an Australian Research Council Future Fellowship. His research interests focus on the development of quantum chemical theory for the calculation of highly accurate chemical properties and the application of these theories to problems of chemical structure, mechanism, and design. Amir's applied studies span several disciplines, ranging from biochemistry to nanochemistry. These include the computational design of 2D functional materials, elucidating the mechanisms by which biomlecules function, and simulations of astrochemically and atmospherically relevant chemical reactions. He has (co-)authored 150 papers, which have been cited over 4,500 times and generate an h-index of 37 (ISI Web of Science). Recent awards include the Le Fèvre Medal from the Australian Academy of Science (2018), the Royal Australian Chemical Institute (RACI) Physical Chemistry Division Lectureship (2019), and the American Chemical Society (ACS) Journal of Physical Chemistry - PHYS Division Lectureship Award (2020). As a scientist, Amir's greatest driving force is the thrill of discovering something new about nature, and, as a research group leader at the University of Western Australia, inspiring students is his ultimate goal. Amir serves as the current Chair of the RACI Inclusion and Diversity Committee (RIDC) and shares his expertise as Editor of Chemical Physics Letters and Associate Editor of the Australian Journal of Chemistry. 